\section{PCSK9 inhibitors are go}

Regulators in the USA and Europe have given the green light to Sanofi and Regeneron's alirocumab, making it the first proprotein convertase subtilisin/kexin type 9 (PCSK9) inhibitor to be approved on both sides of the Atlantic. In the USA, the cholesterol-lowering drug is approved for use in patients with heterozygous familial hypercholesterolaemia and in patients with clinical atherosclerotic cardiovascular disease who require additional lowering of low-density lipoprotein (LDL) cholesterol. In the European Union (EU), the label explicitly includes statin-intolerant patients.

The European Medicines Agency (EMA) also recently approved Amgen's PCSK9-targeting antibody, evolocumab, for use in the EU. As Nature Reviews Drug Discovery went to press, analysts expected the US Food and Drug Administration (FDA) to approve evolocumab by the end of August.

Each antibody is predicted to earn over $\$$ US2 billion by 2020, according to consensus global sales forecasts from Thomson Reuters Cortellis. The drugs are also set to provide a proof of principle for high-volume use of antibodies in a chronic-disease market, although there is pushback at their cost (over $\$ 14,000$ per year for alirocumab in the USA).

PCSK9 binds the LDL receptor on the surface of liver cells, resulting in reduced removal of LDL cholesterol from the blood. By blocking PCSK9, these receptors are free to clear LDL cholesterol from the blood, lowering LDL cholesterol levels. Work on the class began after researchers found a gain-of-function PCSK9 mutation that was associated with hypercholesterolaemia, in 2003. When they subsequently found a loss-of-function mutation that was associated with decreased LDL cholesterol levels and lower risk of coronary artery disease, interest increased. The relatively fast development of the class provides a compelling example of the benefits of genetic validation of drug targets (Nat. Rev. Drug Discov. 12, 581-594; 2013).

Pfizer's PCSK9 inhibitor bococizumab is in Phase III trials, with top-line results expected next year. Pfizer is also developing a small-molecule PCSK9 inhibitor, which it plans to advance into clinical trials later this year, and a vaccine against PCSK9, which it plans to advance into clinical trials next year.

Asher Mullard

\section{Crowdfunding clinical trials}

Is crowdfunding an effective way to raise money for clinical trials? And, if so, are such trials in the best interest of patients, researchers and science? Yes, and not yet, found two recent papers that looked at these questions.

To assess the success rate of crowdfunding clinical research, investigators scoured 14 popular crowdfunding websites, including 4 dedicated solely to medical research, to identify clinical trial fundraising campaigns (Lancet 386, 338; 2015). They found 20 in total, including 7 that were ongoing. Of the 13 completed campaigns, 8 (62\%) had met their fundraising objectives. These campaigns raised from US\$3,600, for a pilot trial looking at the effect of cannabis on attention deficit disorder, to $\$ 3$ million, for a Phase I trial of an oncolytic virus. The average amount raised was $\$ 540,000$, and the median was $\$ 167,000$.

Given decreasing funding from national public agencies, "this strategy might be especially useful for pilot or Phase I studies," the authors write.
But an ethical analysis of "patient-funded trials", which include crowdfunded trials and pay-for-access trials, raised several red flags about this approach (Cell Stem Cell 17. 135-137; 2015). These trials side step scientific peer review, co-opting the 'therapeutic hope' of desperate patients to proceed despite potentially "little or no scientific foundation". A short-term incentive structure for sponsors of crowdfunded trials can lead to poor trial design and low-quality clinical data, the authors also note, and these trials can recruit patients away from studies grounded in stronger scientific rationale.

While crowdfunded trials can "empower patients, expand available research resources and accelerate the pace of translation", the authors of the ethical analysis argue that as yet they harbour "important liabilities for both patients and the broader clinical research enterprise". Funding bodies and research institutions need to establish novel mechanisms to review the merit and design of patient-funded trials if such trials are going to meet the interests of patients, science and society, they write.

Asher Mullard

\section{Ebola vaccine yields first hopes of clinical efficacy}

An interim analysis of a clinical trial of Merck \& Co. and NewLink Genetics' Ebola vaccine showed that the vaccine has so far provided $100 \%$ protection from the deadly haemorrhagic virus. In the "cluster-randomized ring vaccination trial', clinicians identified contacts of confirmed Ebola cases and randomly assigned each cluster to either immediate treatment with rVSV-ZEBOV or to delayed treatment, 21 days later. There were 0 cases of Ebola in the 4,100 people who received immediate vaccination, compared with 16 cases of Ebola in the 3,500 people who received delayed vaccination (Lancet, published online 3 Aug 2015). Further trials are ongoing to determine whether the promising interim findings hold up.

The data also provide proof of concept for the cluster-randomized ring vaccination trial design, which could be useful for the development of vaccines against other viral outbreaks.

Merck's rVSV-ZEBOV consists of a recombinant, replication-competent vesicular stomatitis virus that expresses the glycoprotein of Zaire Ebola virus. GlaxoSmithKline and Johnson \& Johnson also have Ebola vaccines in clinical development.

Asher Mullard

\section{EMA approves first ever malaria vaccine}

The European Medicines Agency (EMA) has approved the first malaria vaccine, GlaxoSmithKline's RTS,S. In placebo-controlled trials in over 15,000 children and infants, the vaccine reduced infection by $36 \%$ in children and by $28 \%$ in infants (Lancet $\mathbf{3 8 6}, 31-45$; 2015). Experts welcomed the landmark approval for what is the first ever vaccine to provide protection from a parasite. But, given the limited protection it affords, they will now have to tackle thorny questions about its cost-effectiveness and public health value. Over 20 antimalarial medicines are also in the clinic, shows a recent review of the evolution of the antimalarial pipeline (Nat. Rev. Drug Discov. 14, 424-442; 2015).

Asher Mullard 